\title{
Growth, Structural and Magnetic Properties of High Coercivity Co/Pt Multilayers
}

\author{
D. WELLER ${ }^{1}$, L. FOLKS, M. BEST, E.E. FULLERTON, B.D. TERRIS \\ IBM Almaden Research Center, 650 Harry Road, San Jose, CA 95120 \\ G.J. KUSINSKI ${ }^{2,3}$, K.M. KRISHNAN ${ }^{2}$, G. THOMAS ${ }^{2,3}$ \\ ${ }^{2)}$ Department of Materials Science and Engineering, University of California, Berkeley, CA 94720, \\ ${ }^{3)}$ Materials Sciences Division, Lawrence Berkeley National Laboratory, CA 94720 ,
}

\begin{abstract}
$\mathrm{Co} / \mathrm{Pt}$ multilayer films $\left(\left[\mathrm{Co}\left(\mathrm{t}_{\mathrm{Co}} \mathrm{nm}\right) / \mathrm{Pt}(1 \mathrm{~nm})\right]_{10}, 0.2<\mathrm{t}_{\mathrm{Co}}<2 \mathrm{~nm}\right)$ with perpendicular magnetic anisotropy and room temperature coercivity $\mathrm{H}_{\mathrm{c}}=12-15 \mathrm{kOe}$ are fabricated using electron beam evaporation at elevated growth temperatures onto $\mathrm{Si}(001) / \mathrm{SiN}_{\mathrm{x}}(40 \mathrm{~nm}) / \mathrm{Pt}(20 \mathrm{~nm})$ substrate/seed structures. Hysteresis and magnetic force microscopy (MFM) studies indicate changes in the magnetization reversal mechanism along with a sharp increase in coercivity for growth temperatures higher $\mathrm{T}_{\mathrm{G}} \sim 230-250{ }^{\circ} \mathrm{C}$. Films grown at $\mathrm{T}_{\mathrm{G}}<230{ }^{\circ} \mathrm{C}$ $\left(\mathrm{t}_{\mathrm{Co}}=0.2-0.4 \mathrm{~nm}\right)$ show large micrometer size magnetic domains and rectangular hysteresis loops indicating magnetization reversal dominated by rapid domain wall motion throughout the film following nucleation at $\mathrm{H}_{\mathrm{n}} \sim \mathrm{H}_{\mathrm{c}}$. Films grown at $\mathrm{T}_{\mathrm{G}}>250{ }^{\circ} \mathrm{C}$ show fine-grained MFM features on the sub-100 nm length scale and hysteresis studies indicate reversal dominated by localized switching of small clusters. The hysteresis curves for the highest coercivity films are sheared with with a hysteresis slope $\alpha=4 \pi \mathrm{dM} / \mathrm{dH} \mid \mathrm{Hc} \cong 1.5$, which is close to the ideal value for completely decoupled grains of $\alpha=1$. High resolution cross-sectional TEM with elemental analysis shows columnar grains extending throughout the multilayer stack. Sharp $\mathrm{Co} / \mathrm{Pt}$ interfaces are found from TEM and grazing incidence X-ray diffraction. At higher $\mathrm{T}_{\mathrm{G}}$, Co depletion and structural defects at the grain boundaries provide a mechanism for exchange decoupling of adjacent grains, which may result in the high coercivities observed. Anisotropy and magnetization values are estimated as $\mathrm{K}_{\mathrm{u}} \sim 8 \times 10^{6} \mathrm{erg} / \mathrm{cc}$ and $\mathrm{M}_{\mathrm{S}} \sim 450 \mathrm{emu} / \mathrm{cc}$ (per total volume), hence $\mathrm{H}_{\mathrm{k}}=2 \mathrm{~K}_{\mathrm{u}} / \mathrm{M}_{\mathrm{S}} \sim 17.5 \mathrm{kOe}$ for the highest coercivity $\mathrm{H}_{\mathrm{c}} \sim 15 \mathrm{kOe}$ films.
\end{abstract}

\section{Introduction}

High coercivity (12 kOe) perpendicularly oriented granular films deposited on magnetically soft underlayers have recently been proposed as media to achieving Terrabit $/ \mathrm{in}^{2}$ areal density in future magnetic recording systems [1]. One of the key requirements is a large ratio of coercivity and magnetization, which in SI units should be about three [2]. Another requirement is a large nucleation field $\left|\mathrm{H}_{\mathrm{n}}\right|$ and optimum shearing of the hysteresis such that the slope at the coercivity is mainly limited by demagnetization: $\alpha=4 \pi \mathrm{dM} /\left.\mathrm{dH}\right|_{\mathrm{Hc}} \cong 1[\mathrm{~B}$. $\mathrm{CoX} / \mathrm{Pt}$ or $\mathrm{CoX} / \mathrm{Pd}(\mathrm{X}=\mathrm{Cr}, \mathrm{B}, \mathrm{Cu}, \mathrm{Ag}, \ldots)$ based multilayers are ideal candidates for this application owing to their large, interface-induced perpendicular magnetic anisotropy and tunability of structural and magnetic properties via growth conditions and Co-layer thickness [ 4$]$ ].

In this paper, we show that growth induced grain boundary decoupling in $\mathrm{Co} / \mathrm{Pt}$ multilayer films can lead to (a) large coercivity and large negative nucleation field, and (b) nearly perfectly sheared loops for recording

\footnotetext{
${ }^{\dagger}$ Now at Seagate Technology LLC, 2403 Sidney Street, Pittsburgh, PA, 15203
} 
applications. Energy filtered TEM studies indicate Co-depletion at the grain boundaries, which is viewed as a possible mechanism for grain decoupling and the large coercivities observed. Key to achieving these properties is high temperature growth $\left(\mathrm{T}_{\mathrm{G}} \sim 300^{\circ} \mathrm{C}\right)$ which, however, has the adverse effect of grain growth. Observed grain diameters in the $30-80 \mathrm{~nm}$ range render the structures in the present study unattractive for perpendicular recording media applications. The present results nonetheless provide significant insight into structural mechanisms leading to high coercivity and exchange decoupling in multilayer films in the absence of chemical segregants.

\section{Sample Preparation}

The Co/Pt multilayer samples under study are fabricated by electron beam evaporation using a $10^{-8}-10^{-9}$ Torr base pressure deposition system. They consist of a $20 \mathrm{~nm} \mathrm{Pt}$ buffer layer, 10 periods of Co $(0.2-2.0 \mathrm{~nm}) / \mathrm{Pt}(1 \mathrm{~nm})$ bilayers, followed by a $1 \mathrm{~nm}$ Pt capping layer. Samples are grown on $\mathrm{SiN}_{\mathrm{x}}$ $(40 \mathrm{~nm})$-coated $\mathrm{Si}(001)$ substrates for magnetic and X-ray diffraction (XRD) studies and on electron transparent $\mathrm{Si}_{3} \mathrm{~N}_{4}$ windows for transmission electron microscopy (TEM) studies (using a Philips CM200FEG). The $\mathrm{SiN}_{\mathrm{x}}$ films are dc-magnetron sputtered and the $\mathrm{SiN}_{\mathrm{x}}$-coated substrates are heated to $\sim 400{ }^{\circ} \mathrm{C}$ before electron beam evaporation of the multilayers at growth temperatures $\mathrm{T}_{\mathrm{G}}=25-350{ }^{\circ} \mathrm{C}$. The Pt buffers, for all samples discussed in this paper, are grown at fixed temperature, $\mathrm{T}_{\mathrm{G}} \cong 350{ }^{\circ} \mathrm{C}$. Growth rates are $\sim 0.05 \mathrm{~nm} / \mathrm{s}$ for $\mathrm{Co}$ and $\mathrm{Pt}$ and the pressure during evaporation is in the low $10^{-7}$ Torr range. Magnetic hysteresis measurements of the as-grown samples are performed using polar and transverse Kerr techniques. MFM measurements are performed with a standard Digital Instrument, Inc. (D3000) microscope and Nanosensors $\mathrm{GmbH} \& \mathrm{Co}$. pyramidal tips coated with CoCr.

\section{Magnetic Properties}

Hysteresis properties of a series of multilayers with fixed nominal Co thickness of $t_{C o}=0.3 \mathrm{~nm}$ were studied as function of the growth temperature in the range $25{ }^{\circ} \mathrm{C}<\mathrm{T}_{\mathrm{G}}<350{ }^{\circ} \mathrm{C}$. Results of perpendicular coercivity measurements of eight such films are shown in Fig. 1 and compared with earlier data from Shiomi et al. [5]. As can be seen, the coercivity increases by more than a factor of two on increasing the $\mathrm{T}_{\mathrm{G}}$ above $\sim 220^{\circ} \mathrm{C}$. This increase in coercivity is accompanied by marked changes in the hysteresis loops as shown in Fig. 2 for $\mathrm{T}_{\mathrm{G}}=210{ }^{\circ} \mathrm{C}$ (lower range) and $\mathrm{T}_{\mathrm{G}}=330{ }^{\circ} \mathrm{C}$ (upper range). In addition, the as-grown domain state is observed via MFM to change from very coarse-structured at low $T_{G}$, to very fine structured at high $T_{G}$, as shown in Fig. 3.

From these data we can surmise the nature of the initial magnetization and demagnetization processes in the films. The low $\mathrm{T}_{\mathrm{G}}$ films exhibit low initial susceptibility in the as-grown state, consistent with a wellpinned domain configuration. When the applied field $\mathrm{H}_{\mathrm{a}}$ reaches a critical field for the domain walls to become unpinned, $\mathrm{H}_{\mathrm{p}} \sim 0.75 \mathrm{H}_{\mathrm{c}}$, there is a rapid increase in magnetization $\mathrm{M}$ until all the walls are annihilated and saturation $M_{s}$ is reached. Upon reversal of the field, we find that the field $H_{n}$ required to nucleate a reverse domain is larger than $\mathrm{H}_{\mathrm{p}}$, and once nucleated, reversal of the entire film occurs very rapidly. These highly rectangular loops with $\mathrm{H}_{\mathrm{n}} \sim \mathrm{H}_{\mathrm{c}}$ may be expected in a structure in which reversal events are communicated throughout the film via strong exchange-coupling between grains. For the high $\mathrm{T}_{\mathrm{G}}$-films, on the other hand, in the as-grown state we see from the MFM data that there is a very fine domain structure, comprising small clusters of similarly oriented grains (interaction domains) with length scales below $100 \mathrm{~nm}$. As a positive field is applied, the magnetization is seen to increase almost linearly with field. From this we surmise that these clusters reverse rather independently of their neighbors (magnetostatic interactions notwithstanding) and over a range of applied fields, consistent with an exchange-decoupled granular system [6]. After the films have reached saturation we find that demagnetization also takes place over a broad range of $\mathrm{H}_{a}$, following onset at a substantial negative field we call $\mathrm{H}_{\mathrm{r}}$. The onset of reversal in the case shown in Fig. 2(b) occurs at 
$\mathrm{H}_{\mathrm{r}} \cong 7.8 \mathrm{kOe}$, and the resultant sheared loop has $\mathrm{H}_{\mathrm{c}}=12.8 \mathrm{kOe}$. The slope of the hsyteresis curve is $\alpha=4 \pi \mathrm{dM} / \mathrm{dH} \mid \mathrm{Hc} \cong 1.5$ for this particular structure.

\section{Structural Characterization}

Figure 4 shows high angle XRD measurements of a series of $\left[\mathrm{Co}\left(\mathrm{t}_{\mathrm{Co}}\right) / \operatorname{Pt}(1 \mathrm{~nm})\right]_{10}$ multilayers with varying Co-layer thickness $\left(0.2 \mathrm{~nm}<\mathrm{t}_{\mathrm{Co}}<2.0 \mathrm{~nm}\right)$ grown at $\mathrm{T}_{\mathrm{G}}=310-330{ }^{\circ} \mathrm{C}$. The high angle data show a large 'stationary' $\mathrm{Pt}(111)$ peak arising from the $20 \mathrm{~nm}$ Pt buffer and the average $\mathrm{CoPt}(111)$ peak due to lattice averaging of $\mathrm{Co}$ and $\mathrm{Pt}$ that shifts to higher angles with increasing Co content. The films are (111) textured with typical rocking curve widths of the $\operatorname{CoPt}(111)$ peak of order $9^{\circ}$, as reported elsewhere [7]. In addition to the $\operatorname{CoPt}(111)$ peak, first order multilayer reflections arising from the periodic structure are observed for all the films. Even the structure with the thinnest Co-layer of nominally one atomic layer $\left(\mathrm{t}_{\mathrm{Co}} \sim 0.2 \mathrm{~nm}\right)$ shows sharp, separated $\operatorname{Pt}(111)$ and $\operatorname{CoPt}(111)$ reflections with sharp multilayer reflections indicating a layered structure with coherent interfaces. A grazing incidence scan of the specular and diffuse X-ray intensity [8] of that film is shown in Figure 4. A clear multilayer peak is not observed in the specular scan. However the diffuse reflectivity, the scattered intensity obtained a few degrees off the specular reflection condition, displays a clear multilayer reflection. The presence of the 'Bragg' peak in the diffuse background but not in the specular scan arises from correlated roughness in the multilayer. The lack of a specular peak indicates that the films are rather rough. This roughness, which increases with growth temperature is also seen in AFM measurements [9]. The roughness is conformal from one layer to the next and the layering still exists on a finite lateral length scale.

Figure 5 shows a TEM cross-section image of a Co $(0.6 \mathrm{~nm}) / \mathrm{Pt}(1 \mathrm{~nm})$ multilayer. The grain growth is typically columnar with grains propagating through the film thickness. High-resolution TEM cross-sectional images show Co to be coherent with the Pt layers for this Co thickness. For increasing Co thickness, coherency loss is observed, and thick Co layers (above $\sim 1.5 \mathrm{~nm}$ ) show hcp stacking. High temperature samples are characterized by increased overall roughness and misalignment of the columns from the normal direction. Figure 5(b) shows a representative HRTEM image of a $\mathrm{Co} / \mathrm{Pt} \mathrm{ML}$ grown at $310{ }^{\circ} \mathrm{C}$. The misalignment of two adjacent columns with respect the surface normal is shown. These two grains misalign by $4^{\circ}$, however larger angles were also observed. For samples grown at lower temperatures this feature is not found. As can be seen from Fig. 5(b), the Co layers appear to be discontinuous across the boundary region. Energy filtered images obtained from a multilayer with larger Co-layer thickness showed similar Co-layer discontinuity at the grain boundary. This result has been discussed in detail elsewhere [10]. Also, we note that bright field TEM images indicate an average grain size of $50 \pm 16 \mathrm{~nm}$.

\section{Conclusions}

High temperature growth of $\mathrm{Co} / \mathrm{Pt}$ multilayers leads to granular films with weakly exchange-coupled grains and large coercivities up to $\mathrm{H}_{\mathrm{c}} \sim 0.8 \mathrm{H}_{\mathrm{K}} \sim 15 \mathrm{kOe}$. Despite growth temperatures of $\mathrm{T}_{\mathrm{G}} \sim 300-350{ }^{\circ} \mathrm{C}$, sharp interfaces inside individual multilayer grains are maintained, explaining the high anisotropies observed. Cobalt depletion and structural defects at the grain boundaries may provide the mechanism for the exchangedecoupling of adjacent grains inferred from magnetic measurements, and may in turn be responsible for the high coercivities observed.

\section{Acknowledgments}

D.W. and L.F. gratefully acknowledge the support by AMRI and DOD/DARPA through Grant No. MDA 972-97-1-003. We thank W. McChesney for $\mathrm{SiN}_{\mathrm{x}}$ deposition and A. Kellock for RBS support. G.K. acknowledges financial support through the "IBM Research Fellowship" program. Work at NCEM at LBNL was supported by the Director, Office of Energy Research, Office of Basic Energy Sciences, Materials Sciences Division of the U.S. Department of Energy under contract No. DE-AC03-76SF00098. 


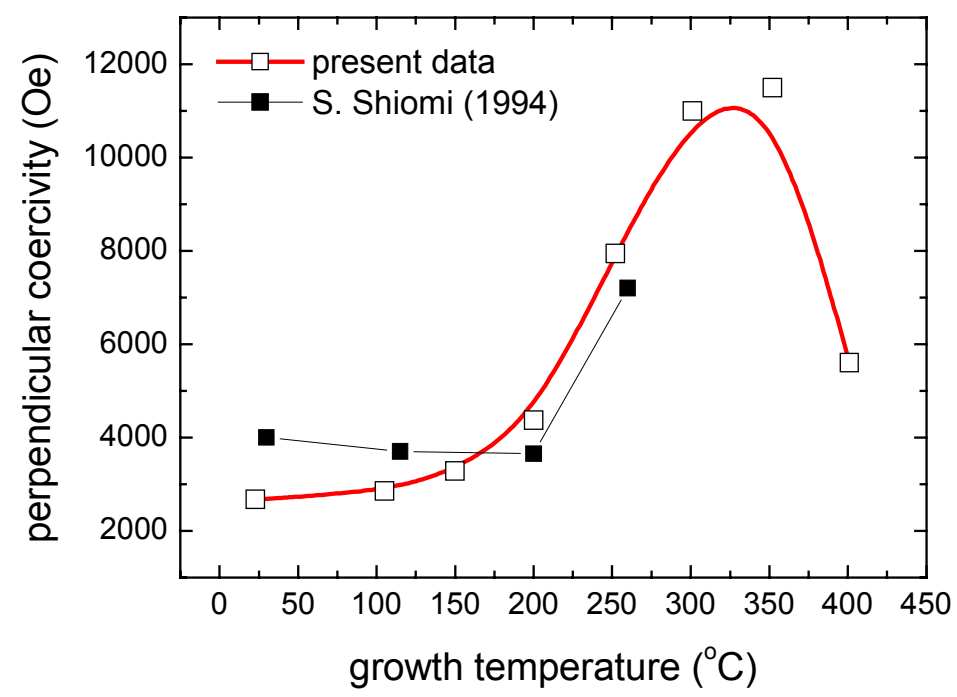

Fig. 1: Growth temperature dependence of the room temperature perpendicular coercivity of a series of $[\mathrm{Co}(0.3 \mathrm{~nm}) / \mathrm{Pt}(1 \mathrm{~nm})]_{10}$ films. Data from Shiomi et al. [5] is given for comparison.
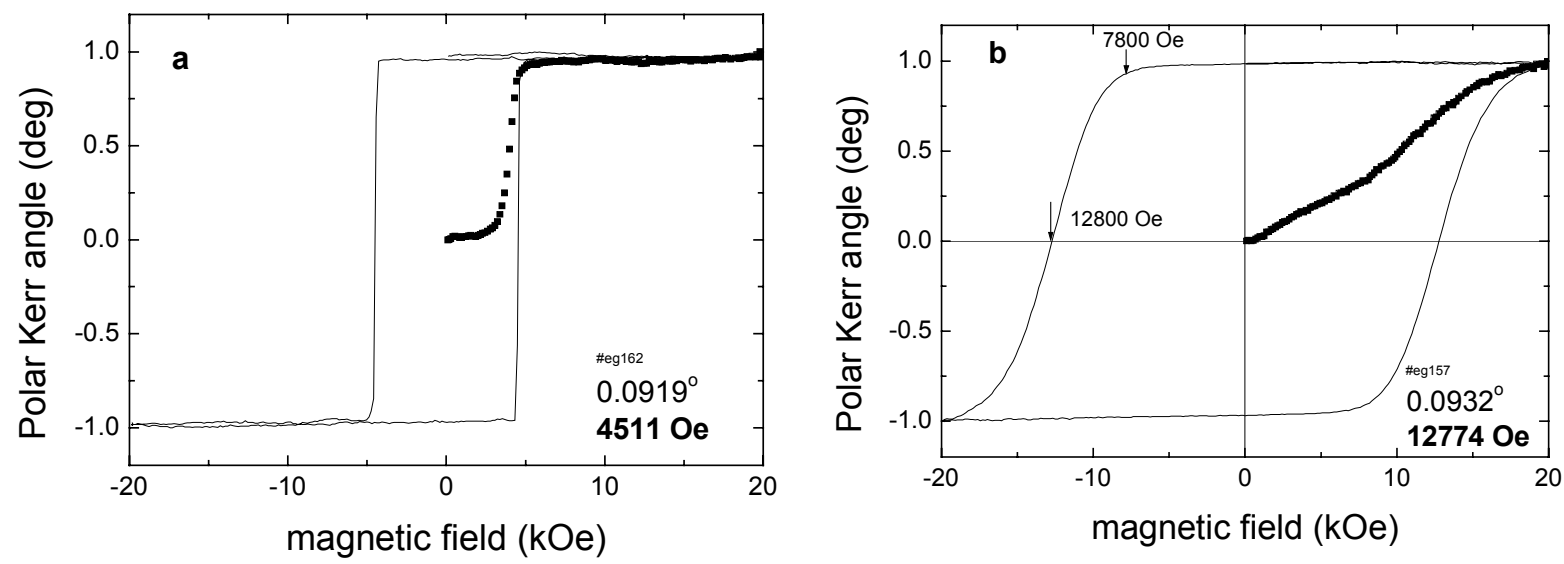

Fig. 2: Polar Kerr initial magnetization and demagnetization data for two $[\mathrm{Co}(0.3 \mathrm{~nm}) / \operatorname{Pt}(1 \mathrm{~nm})]_{10}$ multilayer films evaporated at (a) $\mathrm{T}_{\mathrm{G}} \cong 210{ }^{\circ} \mathrm{C}$ and (b) $\mathrm{T}_{\mathrm{G}} \cong 330{ }^{\circ} \mathrm{C}$; the initial magnetization curves are indicated by the large symbols and the demagnetization curves by the thin lines. 


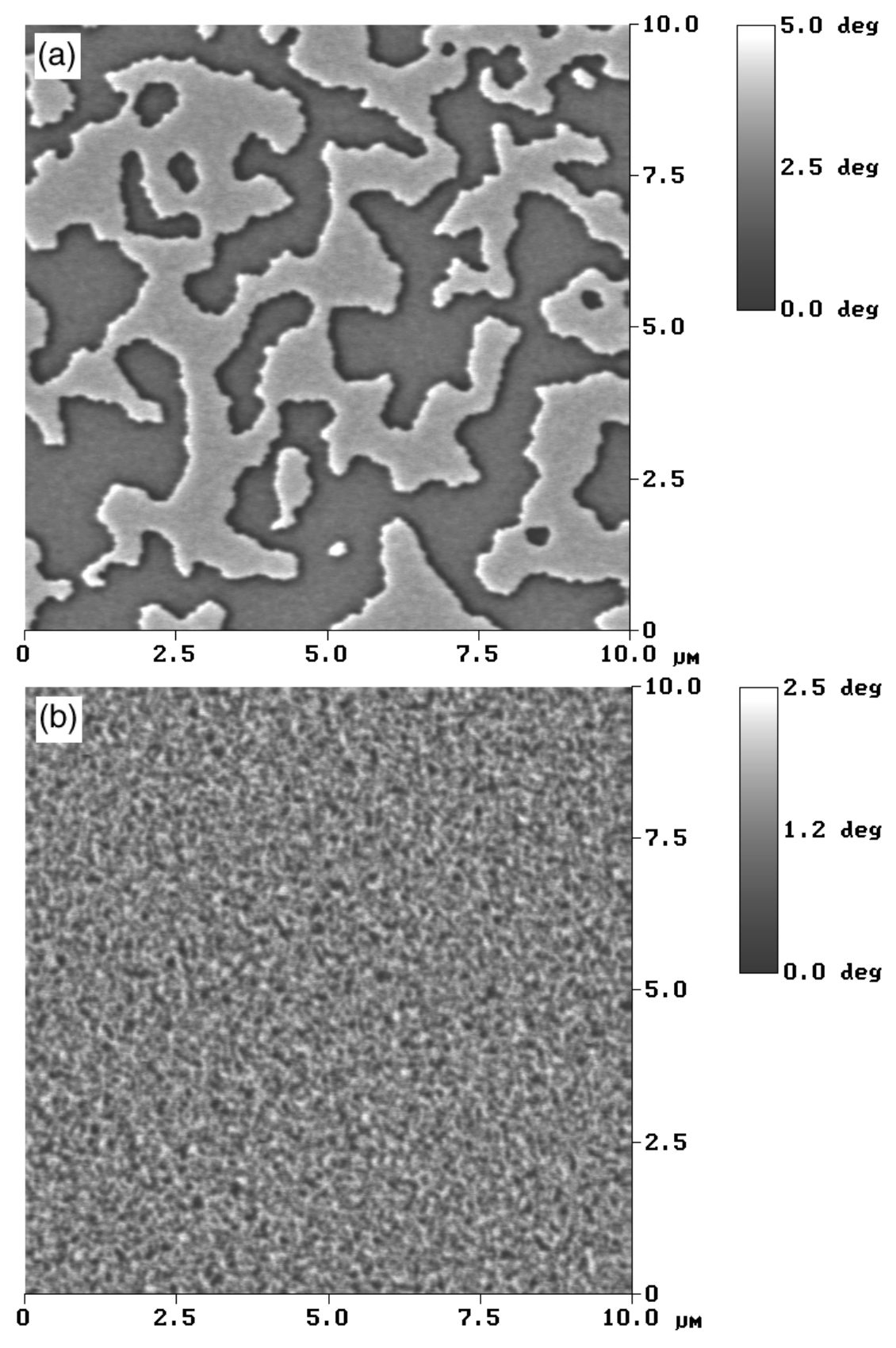

Fig. 3: $10 \times 10 \mu \mathrm{m}$ phase-sensitive MFM images of samples grown at (a) $\mathrm{T}_{\mathrm{G}} \cong 220{ }^{\circ} \mathrm{C}$, and (b) $\mathrm{T}_{\mathrm{G}} \cong 350{ }^{\circ} \mathrm{C}$. 

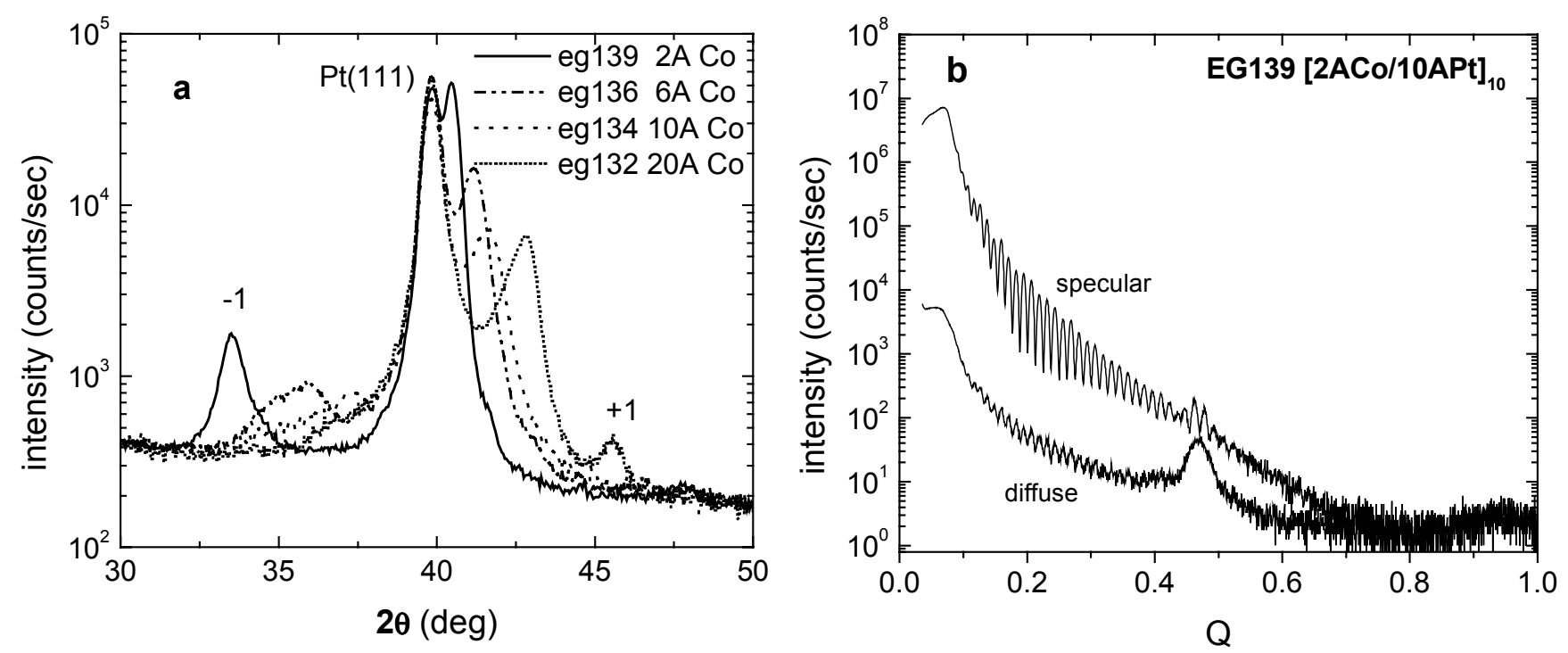

Fig.4: (a) Specular $\theta-2 \theta$ XRD measurements of $\left[\mathrm{Co}\left(\mathrm{t}_{\mathrm{Co}}\right) / \mathrm{Pt}(1 \mathrm{~nm})\right]_{10}$ multilayers grown at $\mathrm{T}_{\mathrm{G}}=310-$ $330{ }^{\circ} \mathrm{C}$ on $20 \mathrm{~nm}$ Pt buffers with $\left(0.2 \mathrm{~nm}<\mathrm{t}_{\mathrm{Co}}<2 \mathrm{~nm}\right)$, and (b) specular and off specular (diffuse) X-ray reflectivity spectra of the $\mathrm{t}_{\mathrm{Co}}=0.2 \mathrm{~nm}$ sample.
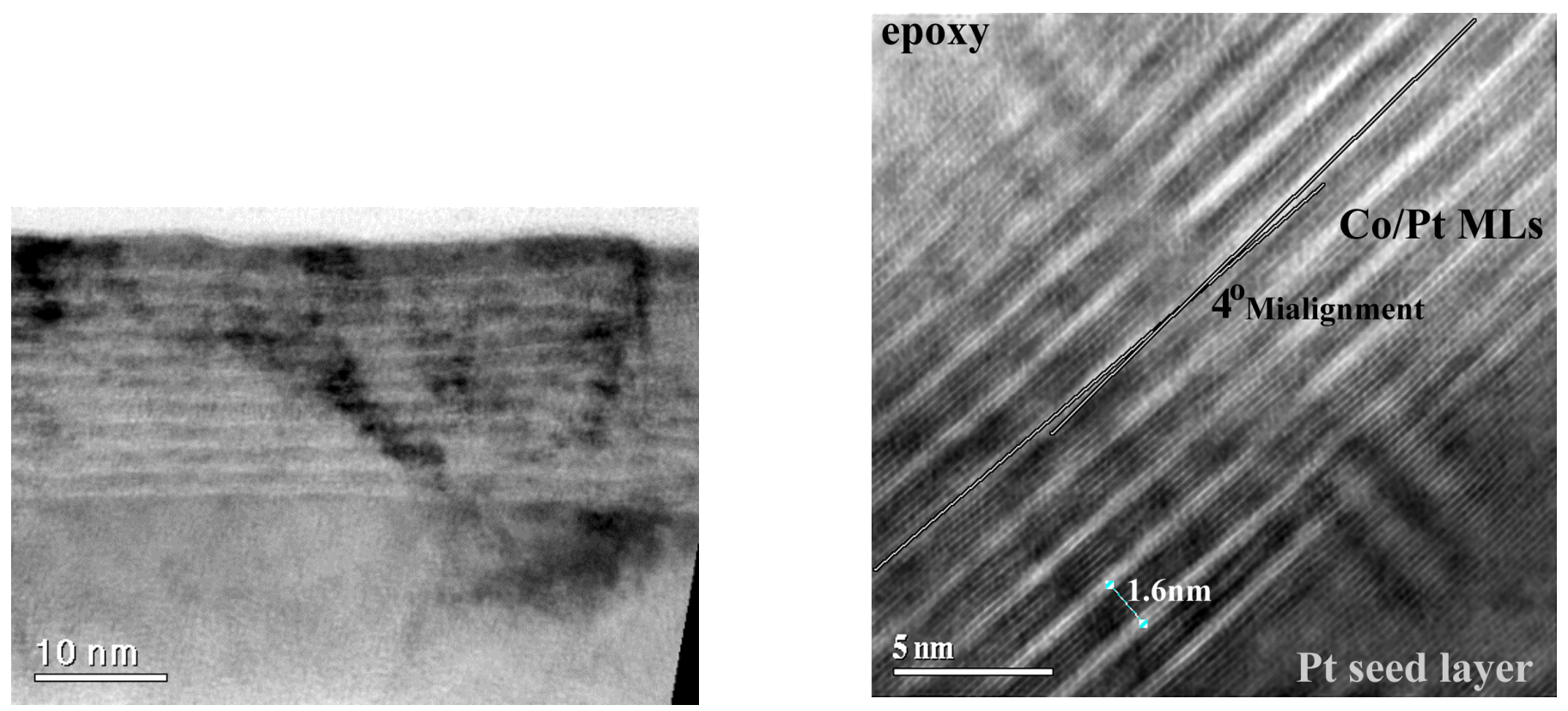

Fig.5: Cross-sectional TEM images of a $[\mathrm{Co}(0.6 \mathrm{~nm}) / \mathrm{Pt}(1 \mathrm{~nm})]_{10}$ multilayer grown at $\mathrm{T}_{\mathrm{G}}=310{ }^{\circ} \mathrm{C}$. 


\section{-References}

1 R. Wood, IEEE Trans. Magn., 36, 36 (2000).

2 H.N. Bertram and M. Williams, IEEE Trans. Magn., 36, 4 (2000).

3 I. Tagawa, A. Takeo and Y. Nakamura, J. Magn. Magn. Mater., 155341 (1996).

4 See e.g. E.E. Fullerton, K. Takano, G. Zeltzer, H. Do, M. Xiao, K. Rubin and J. Kortright, PMRC 2000, October 23-26, 2000, Sendai, Japan

5 S. Shiomi et al., Trans. Mat. Res. Soc. Jpn., 15B, (1994).

6 T. Suzuki, H. Notarys, D.C. Dobbertin, C.-J. Lin, D. Weller, D. C. Miller, G. Gorman, IEEE Trans. Magn. 28, 2754 (1992).

7 D. Weller et al., J. Appl. Phys., 87, 5768 (2000).

8 V. Holy, U. Pietsch, T. Baumbach, "High-Resolution X-ray Scattering from Thin Films and Multilayers", Springer, Berlin, 1999.

9 The AFM rms roughness determined from $0.5 \times 0.5 \mu \mathrm{m}$ scans increases sharply from a plateau of about $0.75 \mathrm{~nm}$ for $\mathrm{T}_{\mathrm{G}}<250{ }^{\circ} \mathrm{C}$ to $>2 \mathrm{~nm}$ for $\mathrm{T}_{\mathrm{G}}>250{ }^{\circ} \mathrm{C}$. Films grown at $\mathrm{T}_{\mathrm{G}} \cong 250{ }^{\circ} \mathrm{C}$ showed an average rms roughness of $1-1.5 \mathrm{~nm}$.

10 G.J. Kusinski, K.M. Krishnan, D. Weller, B.D. Terris, L. Folks, A.J. Kellock, J.E.E. Baglin, and G. Thomas, "Lorentz transmission electron microscopy investigation of magnetically patterned $\mathrm{Co} / \mathrm{Pt}$ multilayers", Conf. Proceedings of Magnetic Storage Systems Beyond 2000, NATO-ASI, 12-23 June 2000, Rhodes, Greece;

G. J. Kusinski (to be published). 